\title{
NOSTOS experiment and new trends in rare event detection
}

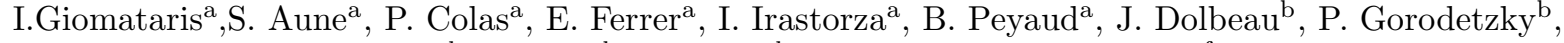

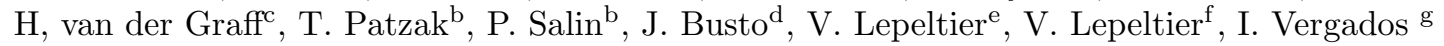 \\ ${ }^{a}$ CEA, Saclay, DAPNIA, Gif-sur-Yvette, Cedex,France. \\ ${ }^{\mathrm{b}}$ IN2P3/CNRS PCC-Collège de France 75231 Paris Cedex 05 France \\ ${ }^{\mathrm{c} N I K H E F}$, Amsterdam The Netherlands \\ ${ }^{\mathrm{d}}$ CCPM-Faculté des Sciences de Luminy, 13288 Marseille Cedex 09 France
}

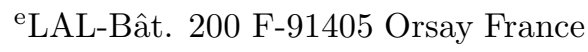

${ }^{\mathrm{f}}$ LBNL Berkeley USA

' University of Ioannina T.P.D., PO Box 1186, 45110 Ioannina Greece

\begin{abstract}
A novel low-energy neutrino-oscillation experiment NOSTOS, combining a strong tritium source and a high pressure spherical TPC detector (10 $\mathrm{m}$ in radius) has been recently proposed. The goal of the experiment is to measure the mixing angle $\theta_{13}$, the neutrino magnetic moment and the Weinberg angle at low energy. The same apparatus, filled with high pressure Xenon, exhibits a high sensitivity as a Super Nova neutrino detector with extra galactic sensitivity. Results of a first prototype will be shown and a short-term experimental program will be discussed.
\end{abstract}

\section{Introduction}

Nowadays there is a very strong evidence for neutrino oscillation from atmospheric and solar neutrino experiments. Recent results from the KamLAND confirm earlier work at the Sudbury Neutrino Observatory and Super-Kamiokande that also provided strong evidence for neutrino oscillation. The fact that different neutrino types do change their identity as they propagate, suggests that they are not strictly massless as had been assumed by the standard weak interaction model. The atmospheric neutrino oscillation data suggest that there is maximal mixing between the $\tau$ and e- neutrinos and a corresponding mass squared difference of $\delta m_{23}^{2}=3 \times 10^{-3} \mathrm{eV}^{2}$. On the other hand the solar neutrino and other electron neutrino disappearance experiments suggest a non maximal mixing of and a mass squared difference of $\delta m_{21}^{2}=7 \times 10^{-5} \mathrm{eV}^{2}$. The other mixing angle is not known but it is quite small constrained from the $\mathrm{CHOOZ}$ data. If one includes this small mixing angle, one can see in electron neutrino experiments the effect of the large $\delta m_{23}^{2}$, i.e. a transition probability associated with the small oscillation length. In other words for a detector close to the source one will see a disappearance oscillation probability of the form:

$P\left(\nu_{e} \rightarrow \nu_{\mu, \tau}\right)=\sin ^{2} 2 \theta_{13} \sin ^{2} \pi \frac{L}{L_{23}}$

where $\mathrm{L}$ is the distance between source and observed interaction, $\mathrm{E}$ is the neutrino energy and $\mathrm{L}_{23}$ is related to the neutrino energy: $L_{23}=$ $2 \pi E_{\nu} /\left(\delta m_{23}^{2}\right)$. In the case of very low energy neutrinos, such as the ones emitted by a tritium source, the oscillation length $\mathrm{L}_{23}$ is only $13 \mathrm{~m}$. Therefore by observing neutrino interactions inside a large TPC of about $10 \mathrm{~m}$ in radius, surrounding a tritium source, one can contain the oscillation occurring inside the gas volume and measure the oscillation parameters by a single experiment. The idea of combining a strong tritium source and a spherical detector has been recently 
proposed 1. In this proposal a large spherical drift volume filled with a suitable gas mixture at high pressures is used for detecting low energy recoils produced in neutrino interactions.

\section{The spherical TPC concept}

The tritium source is located in a small spherical vessel of about $25 \mathrm{~cm}$ radius and it is surrounded by a spherical gaseous TPC $10 \mathrm{~m}$ in radius as shown in figure 1. Low energy electron recoils produced in the gas by elastic scattering in the TPC volume are ionizing the gas. Charges are drifting towards the sphere center and are collected by an adequate gaseous detector. The use of a spherical Micromegas counter made out of flat detectors was advertised because of the high precision and excellent energy resolution 23. The high efficiency for detecting single electrons has been demonstated [4]even at high pressures [5]. The detector is currently used for solar axion detection in the CAST experiment [6] where a great stability and ability to reject background events has been achieved.

The novel approach is radically different from all other neutrino oscillation experiments in that the neutrino source and the detector are located in the same vessel; it is then possible to measure the neutrino interactions, as a function of the distance source-interaction point, with an oscillation length that is fully contained in the detector. Indeed we expect a counting rate oscillating from the centre of the sphere towards the external volume, i.e. at first a decrease, then a minimum and finally an increase. In other words we will have a full observation of the oscillation process as it has already been done in accelerator experiments with neutral strange particles. Fitting such an observed curve will provide all the relevant parameter of the oscillation by a single experiment. It is equivalent to many experiments made in the conventional way where the neutrino flux is measured in a single space point. The use of a spherical TPC detection scheme presents many advantages :

- It is a natural focus device requiring a small amplifying detector with only a few readout channels. Such small size detector sim- plifies the construction and reduces the cost of the project.

- With the neutrino source at the center of curvature the detected signal is optimized with a gain of a significant factor compared to the signal provided by a cylindrical TPC with the detector aside the end-cup of the TPC.

The electric field at a distance $r$ from the center of curvature, in the case of a spherical TPC, is given by :

$E=\frac{V_{0}}{r^{2}} \frac{R_{1} R_{2}}{R_{2}-R_{1}}$

where $R_{1}$ and $R_{2}$ are the internal and outer sphere radii and $\mathrm{V}_{0}$ is the applied voltage. At low electric fields (E) the drift velocity $v_{D} \approx E$ is roughly proportional to $\mathrm{E}$ and the longitudinal diffusion coefficient is $D \approx 1 / \sqrt{E}$, inversely proportional to the square of E. From the previous expressions one can easily deduce that time dispersion is :

$\sigma_{t}=\frac{\sigma_{L}}{v_{d}}=\frac{D \sqrt{r}}{v_{d}} \approx \frac{1}{E^{3 / 2}} \approx r^{3}$

From the latter equation one can conclude that in the spherical detector there is enhancement of the time dispersion of the detected signal especially at large distances. We propose to use this property for estimating the depth of the produced electron recoil during the neutrino elastic scattering. First calculations show that a precision for the depth of the interaction point of better than $10 \mathrm{~cm}$ will be achieved by measuring the time dispersion of drifting charges. Using a $20 \mathrm{Kg}$ tritium source, the total rate of emitted neutrino is $6 \times 10^{18} / \mathrm{s}$. With a gas filling of Xe at $\mathrm{p}=1$ bar the rate of detected neutrino is about 1000 /year. We are studying various issues for increasing the pressure in Xenon or other noble gases in order to get a larger signal or decrease the amount of the tritium source.

\section{Sensitivity for the neutrino magnetic moment}

Because of the low energy of the incoming neutrinos and the low energy electron recoils detected 
in this experiment the sensitivity for the neutrino magnetic moment is high. The cross section of the magnetic moment can be written as :

$$
\left(\frac{d \sigma}{d T}\right)_{E M}=\sigma_{0}\left(\frac{\mu_{l}}{10^{-12} \mu_{B}}\right)^{2} \frac{1}{T}\left(1-\frac{T}{E_{\nu}}\right)
$$

Because of the dependance $1 / \mathrm{T}$ ( $\mathrm{T}$ is the electron recoil energy) the sensitivity for the magnetic moment is obviously higher at low energy. Indeed precise calculations show that the differential cross section due to a neutrino magnetic moment of $10^{-12} \mu_{B}$ is rising at low energy and reaches $30 \%$ of the value of the weak neutrinoelectron cross section which is at a first order flat with the recoil energy. We would like to point out that recent measurements from the NuMu experiment predict a limit of $10^{-10} \mu_{B}$ for the neutrino magnetic moment. Our experiment opens the way to improve this value by two orders of magnitude.

\section{The measurement of the weak charge}

Another interesting quantity is the Weinberg angle appearing in $\sin ^{2} \theta$, which is a function of the momentum transfer and it has not been measured at such low transfers. To this end atomic physics experiments, which utilize the neutral current, have thus far been considered. The neutral current at low energies gives a current-current interaction of the form:

$$
\begin{aligned}
H_{W}= & \frac{G_{F}}{\sqrt{2}} J_{\lambda}^{Z} J_{Z}^{\lambda} \\
J_{\lambda}^{Z}= & \bar{p} \gamma_{\lambda}\left(1-4 \sin ^{2} \theta_{W}-\gamma_{5}\right) p+ \\
& +\bar{n} \gamma_{\lambda}\left(1-\gamma_{5}\right) n- \\
& -\bar{e} \gamma_{\lambda}\left(1-4 \sin ^{2} \theta_{W}-\gamma_{5}\right) e \ldots
\end{aligned}
$$

Thus the atomic physics experiments suffer from the fact that the weak charges involved are extremely small, i.e. the weak charge of the electron is $-1+4 \sin ^{2} \theta=-0.1$, while for the proton is 0.1 . Due to this smallness one has to deal with complications in the analysis of the experiments arising from radiative corrections. This has also implications in the neutrino nucleus elastic scattering in the sense that the neutrons in the nucleus can contribute coherently. The coherence due to the protons is suppressed by the smallness of the weak charge. In the proposed experiment such cancellations do not occur and one needs not worry about such corrections. Furthermore by plotting the differential neutrino-electron cross section as a function of the electron energy we obtain a straight line. We hope to construct the straight line quite accurately. Thus we can extract a value of the Weinberg angle both from the slope and the intercept achieving high precision.

\section{Supernova sensitivity}

It is generally believed that the core-collapse supernova explosion produces a large number of neutrinos and $99 \%$ of the gravitational energy is transformed to neutrinos of all types. The supernova $(\mathrm{SN})$ neutrino flux consists of two main components: a very short $(<10 \mathrm{msec})$ pulse of $\nu_{e}$ produced in the process of neutronization of the SN matter through the reaciont $\mathrm{e}+\mathrm{p} \rightarrow \mathrm{e}+$ $\mathrm{n}$, which is followed by a longer $(<10 \mathrm{sec})$ pulse of thermally produced $\nu_{e}, \nu_{\mu}, \nu_{\tau}$, and their antiparticles. Only a small fraction, about $1 \%$, of the neutrinos are prompt, while the rest are neutrino pairs from later cooling reactions. Therefore the neutrino signal from a supernova rises first steeply and then falls exponentially with time in a time window of about 10 seconds. It is expected that spectra of thermally produced neutrinos are characterized by the different mean energies : $\nu_{e}=11 \mathrm{MeV}, \bar{\nu}_{e}=16 \mathrm{MeV}, \nu_{e, \mu}=25 \mathrm{MeV}$. Our idea is to use the large cross section offered by the coherent neutrino-nucleus cross section for detecting neutrinos from Super Nova explosions. Coherent scattering occurs when neutrinos interact with two or more particles and the amplitudes from the various constituants of the target adding up. A consequence of the coherence is an increase of the cross section becoming proportional to the square of the number of particles in the target leading to ncreased counting rates :

$\sigma=\frac{G^{2} N^{2} E^{2}}{4 \pi}$

where $\mathrm{G}$ is the weak coupling constant, $\mathrm{N}$ is the number of neutrons in the target nucleus and $\mathrm{E}$ 
is the neutrino energy. In order to get advantage of the coherent scattering amplification of heavy nuclei gases are needed.

For instance, using Xenon as detector target the coherent cross sections at $\mathrm{E}=25 \mathrm{MeV}$, the energy that is relevant for Supernova detection, is quite large $\left(\sigma=1.510^{-38} \mathrm{~cm}^{2}\right)$. Even at lower energy $(11 \mathrm{MeV})$ where the coherent cross sections decreases quadratically with energy, the cross section is still high, in the case of gaseous Xenon detector. The recoil energy energy is quite low and it takes a maximum value of $1.5 \mathrm{keV}$ for 11 $\mathrm{MeV}$ and $9 \mathrm{keV}$ for $25 \mathrm{MeV}$ neutrinos. This implies that detector thresholds must be set quite low with one advantage that backgrounds are highly suppressed given the narrow time window in which the burst takes place. The collected energy is even lower by a significant factor (quenching factor) and therefore sub-keV detector threshold is required as it is the case of the NOSTOS spherical TPC. For a typical galactic SN explosion the detector used for the tritium experiment ( $10 \mathrm{~m}$ in radius, $\mathrm{p}=10$ bar of Xenon) the number of detected neutrinos will exceed 100,000. Such high signal will also permit extra galactic detection. A possibility to test the efficiency of detecting coherent neutrino scattering will be the nuclear reactor. The expected number of neutrino interactions in the gas volume, from a typical reactor neutrino flux and spectrum $\left(10^{13} \mathrm{~cm}^{-2}\right.$ $\mathrm{s}^{-1}$ ) using a detector filled with Xenon is about $350 /$ day $/ \mathrm{Kg}$. The drawback is the very low energy threshold needed since the maximal recoil energy is $185 \mathrm{eV}$. Therefore single electron counting is required imposing a high gain operation of the detector and a measurement of the quenching factor of the ionization produced by the low energy recoils. Such an interesting measurement could be possible by using the first prototype that is described in the next section.

\section{The 1st spherical prototype and results with a new proportional counter}

We have built a spherical prototype $1.3 \mathrm{~m}$ in diameter as a first step towards the large detector needed for accomplishing the NOSTOS project. A schematic view of the prototype is shown in figure 2. The outer diameter is made of pure $\mathrm{Cu}$ (6 $\mathrm{mm}$ thick) alowing to reach pressures as high as 5 bar. The $1 \mathrm{~m} 3$ gaseous volume is pumped through a primary followed by a turbo molecular pump. The quality of the various materials assures a good vacuum $\left(<10^{-6}\right.$ mbar $)$ with a quite low level of outgasing that has been measured to be $<10^{-9} \mathrm{mbar} / \mathrm{s}$. Such low outgasing give us the possibility to avoid a permanent gas circulation through special cleaning filters. First tests were performed by filling the volume with pure gas (argon $+10 \% \mathrm{CO}_{2}<3 \mathrm{ppm}$ impurities) and operate the detector in a seal mode. We are proposing the use of Micromegas as amplifying structure. A particular effort is actually made to build a spherical Micromegas detector using new technologies. Another alternative will be to approximate a sphere by using several Micromegas flat elements. Preparing an ideal solution for the amplification structure we decided to start the first tests by using a small sphere $(10 \mathrm{~mm}$ in diameter made of steel) as a proportional counter located at the center of curvature of the TPC. Several tests from low pressure to high pressure have shown that such a simple amplification element is able to provide high gains and stable operation. With outer sphere at ground and the inner sphere at the High Voltage (HV), signals produced by cosmic rays or a ${ }^{109} \mathrm{Cd}$ radioactive source have been observed at various pressures. A typical signal from the cadmium source is shown in figure 3 . It has a rise time of about $2 \mu$ s that is due to the movement of positive ions. This is quite a long rise time but this simple structure will allow us to make fast progress, understand the functioning of the spherical TPC and accomplish part of the short term experimental program to establish the new concept that can be summarized as follows:

- Tests of the 1st prototype and optimization of the amplification structure.

- Optimize the detector for very-high gain operation.

- Measure the attenuation length of drifting electrons.

- Optimize the energy resolution. 
- Measure the accuracy of the depth measurement by the time dispersion of the signal.

- Optimize mechanics and electronics, use low-radioactivity materials.

- Improve the simulation program.

- Calculate (or measure) the quenching factor in various gases (Xe, Ar..).

- Measure the coherent neutrino scattering next to a reactor.

\section{Conclusions}

An ambitious experimental program in the low energy neutrino physics sector is proposed. It includes observation of neutrino oscillations, neutrino magnetic moment and Weinberg angle at low energy measurement. The same device exhibits a high sensitivity super Nova neutrino detection with extragalactic sensitivity. The measurement of the coherent neutrino nucleus scattering will open the way to build simple, low cost and robust telescopes dedicated for super Nova neutrino detection. A first prototype $1.3 \mathrm{~m}$ in diameter has been build giving first promising results. A novel concept of spherical proportional counter has been successfully tested.

\section{REFERENCES}

1. Y. Giomataris and J. D. Vergados, arXiv:hep-ex/0303045

2. Y. Giomataris, P. Rebourgeard, J. P. Robert and G. Charpak, Nucl. Instrum. Meth. A 376 (1996) 29.

3. Y. Giomataris, Nucl. Instrum. Meth. A 419 (1998) 239.

4. J. Derre, Y. Giomataris, P. Rebourgeard, H. Zaccone, J. P. Perroud and G. Charpak, Nucl. Instrum. Meth. A 449 (2000) 314.

5. P. Gorodetzky et al., Nucl. Instrum. Meth. A 433 (1999) 554.

6. J. I. Collar and Y. Giomataris, Nucl. Instrum. Meth. A $\mathbf{4 6 9}$ (2001) 249 arXiv:hep-ex/0009063.

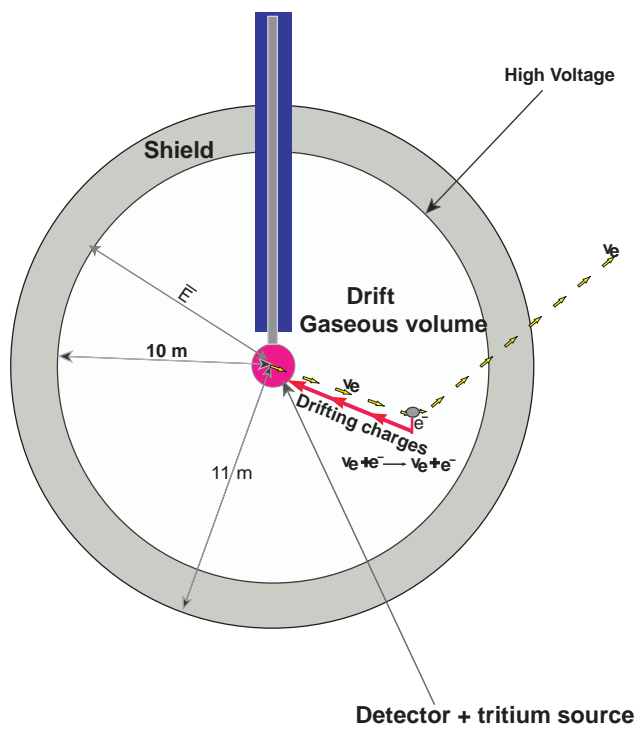

Figure 1. Scheme of the NOSTOS detector

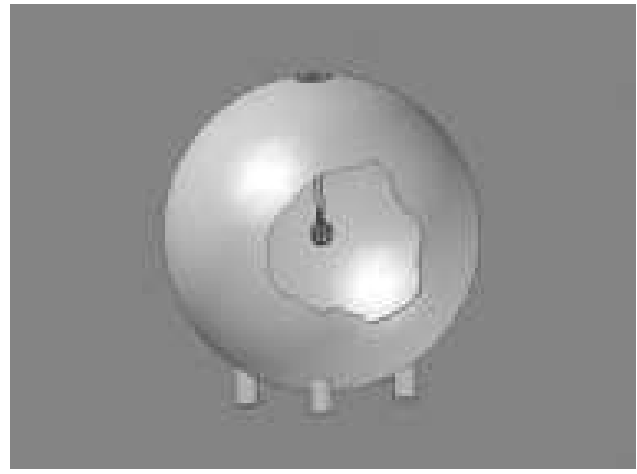

Figure 2. Scheme of the NOSTOS detector 


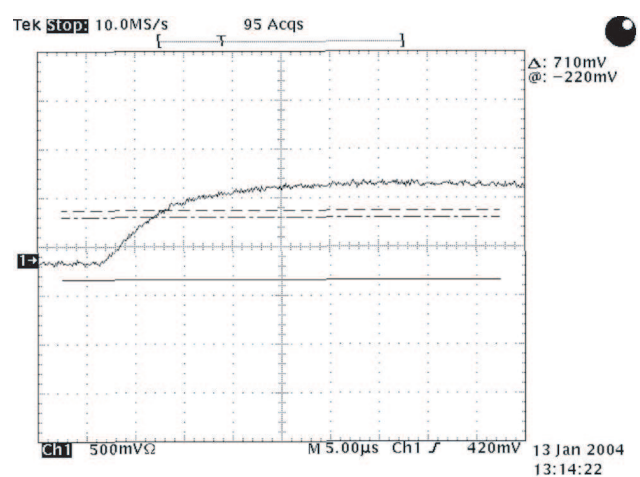

Figure 3. A signal from the $109 \mathrm{Cd}$ radioactive source with the detector filled with $\mathrm{Ar}+10 \% \mathrm{CO}_{2}$ at $\mathrm{P}=600 \mathrm{mbar}$. The long tail is due to the large $\mathrm{RC}$ of the charge amplifier.

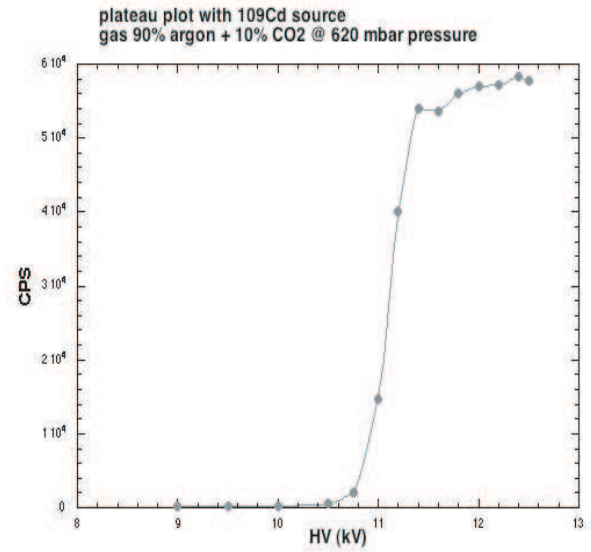

Figure 4. Counting rate versus high voltage using a $109 \mathrm{Cd}$ radioactive source. 